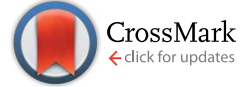

Cite this: RSC Adv., 2017, 7, 16290

Received 5th February 2017

Accepted 7th March 2017

DOI: $10.1039 / \mathrm{c} 7 \mathrm{ra01474k}$

rsc.li/rsc-advances

\section{Aptamer-based fluorescent detection of ochratoxin A by quenching of gold nanoparticles $\uparrow$}



A simple, rapid, low cost and highly sensitive method for the detection of ochratoxin A (OTA) was developed based on the principle that dispersed AuNPs show a better fluorescence quenching effect than aggregated AuNPs. In the absence of OTA, the aptamer is adsorbed onto the surface of AuNPs, which helps to enhance the stability of AuNPs against salt-induced aggregation, and also enhances the fluorescence quenching of the fluorescein-labeled aptamer. With the addition of OTA, the conformation of the aptamer changed, which induced aggregation of AuNPs in the presence of high-salt conditions. The fluorescence intensity was clearly recovered. The assay showed a linear response toward OTA concentration in the range of $25 \mathrm{nM}$ to $300 \mathrm{nM}$ with a correlation coefficient of 0.9957 . The limit of detection for OTA was experimentally determined to be $22.7 \mathrm{nM}$. This method has the advantages of simple operation, low-cost and high sensitivity compared to conventional methods and can be applied to the detection of real samples.
\end{abstract}

\section{Introduction}

Ochratoxin A (OTA) is a highly potent mycotoxin, which has documented nephrotoxic, teratogenic and immune toxicity effects in humans. As a food contaminant, OTA is often found in a wide variety of agricultural produce and food products. ${ }^{1-3}$ At present, OTA residues are restricted in many countries. In China, the allowed concentration of OTA is lower than $100 \mathrm{mg}$ $\mathrm{kg}^{-1}$ according to the National Standard. ${ }^{4}$ Many methods have been used to detect OTA including high-performance liquid chromatography (HPLC), ${ }^{5,6}$ thin layer chromatography (TLC) ${ }^{7}$ and enzyme linked immunosorbent assay (ELISA). ${ }^{6,8,9}$ However, these traditional methods are rather expensive, time consuming and have to be performed by qualified staff only in specialized laboratories. In addition, these methods usually require organic solvents to extract toxins from food, which creates additional pollution for the environment. ELISA is the main method used for the determination of OTA as it is a rapid detection technique. However, the disadvantage of the ELISA method is the necessity of using enzyme-labeling reagents, which are expensive. Moreover, environmental conditions such as temperature, $\mathrm{pH}$, etc. have a significant effect on antibodies or antigens. It is also difficult to use ELISA to detect OTA in the field or on-site. ${ }^{10}$ Therefore, there is an urgent requirement for direct, rapid, and low cost methods for the detection of OTA.

${ }^{a}$ Shandong Provincial Key Laboratory of Chemical Energy Storage and Novel Cell Technology, School of Chemistry and Chemical Engineering, Liaocheng University, Liaocheng 252059, China. E-mail: shuhaowang@sohu.com; Fax: +86 635 8239227; Tel: +866358239227

${ }^{b}$ College of Life Science, Liaocheng University, Liaocheng 252059, China

$\dagger$ Electronic supplementary information (ESI) available. See DOI: $10.1039 / \mathrm{c} 7 \mathrm{ra} 01474 \mathrm{k}$
Gold nanoparticles (AuNPs) have drawn much attention due to their unique properties. ${ }^{11,12}$ In particular, the surface plasma resonance properties (SPR) of AuNPs make them very attractive in colorimetric analysis. ${ }^{13}$ However, colorimetric analysis has low sensitivity and suffers from interference by the background color. In many fluorescence-based methods, AuNPs have been labeled and used as the quenchers. ${ }^{14,15}$ However, complicated surface-chemical modifications of AuNPs can reduce their sensitivity and selectivity, and thus limit their practical application. To overcome these problems, label-free fluorescence detection $^{16,17}$ has been developed to replace traditional methods, which avoids the frequent modification and separation. ${ }^{18,19}$ With the advantages of simple operation, high sensitivity and low cost, this approach has the potential to be widely used in medical analysis.

In this study, a new fluorescent method was developed based on the fact that dispersed AuNPs show better fluorescence quenching effect than aggregated AuNPs. ${ }^{20}$ In the absence of OTA, FAM (carboxyfluorescein) modified aptamer gets absorbed onto the surface of AuNPs preventing the aggregation of AuNPs, and the fluorescence can be quenched by AuNPs. In the presence of OTA, FAM modified aptamer was not absorbed onto the surface of AuNPs, resulting in the aggregation of AuNPs in NaCl solution. As a result, the fluorescence of FAM modified aptamer increased, and further caused the obvious recovery of the fluorescence of FAM modified aptamer. Obviously, common steps could be effectively avoided, such as modifying aptamers onto the AuNPs, complicated centrifugation and separation. As a result, OTA at concentration as low as $22.7 \mathrm{nM}$ can be detected with this method. This method has several advantages including low cost, simple operation and high sensitivity, making it highly useful for the on-site detection of OTA. 


\section{Experimental}

\subsection{Material and reagents}

OTA was purchased from Pribolab Pte. Ltd. (Singapore, http:// www.pribolab.com). The sequence of fluorescein (FAM)labeled anti-OTA aptamer was FAM-5'-GAT CGG GTG TGG GTG GCG TAA AGG GAG CAT CGG ACA (FAM-Apt). The FAM-Apt was synthesized by Shanghai Sangon Biotechnology Co. Ltd. (Shanghai, China, http://www.sangon.com/). Chloroauric acid $\left(\mathrm{HAuCl}_{4}\right)$ was obtained from Sigma-Aldrich (St. Louis, MO, USA, http://www.sigmaaldrich.com/sigma-aldrich/home.html).

Other chemicals, including sodium citrate, sodium chloride, dipotassium hydrogen phosphate, potassium dihydrogen phosphate and magnesium chloride were purchased from Tianjin Chemical Reagent Company (Tianjin, China, http:// www.b2b168.com/c168-143645.html). All chemicals were of analytical grade. Millipore Milli-Q water $\left(18 \mathrm{M} \Omega \mathrm{cm}^{-1}\right)$ was used in all experiments. Phosphate buffer (PB) was composed of $10 \mathrm{mM} \mathrm{KH}_{2} \mathrm{PO}_{4}-\mathrm{K}_{2} \mathrm{HPO}_{4}$ and $1 \mathrm{mM} \mathrm{MgCl}_{2}, \mathrm{pH}=8.5$.

\subsection{Apparatus}

UV visible absorption spectra were recorded on a Shimadzu UV$2550 \mathrm{UV} / \mathrm{Vis}$ spectrophotometer (Kyoto, Japan). All fluorescence intensity measurements were performed on an Infinite M200 multi-detection microplate reader (Tecan, Switzerland). The excitation wavelength was $475 \mathrm{~nm}$, and the spectral region between $500 \mathrm{~nm}$ and $600 \mathrm{~nm}$ was monitored.

\subsection{Preparation of AuNPs}

All glassware used in the experiments was soaked in aqua regia overnight before the preparation of AuNPs. We adopted the method of sodium citrate reduction of chloroauric acid to prepare the AuNPs. In a typical procedure, $50 \mathrm{~mL}$ of $0.04 \% \mathrm{HAuCl}_{4}$ was heated to a rolling boil in a $250 \mathrm{~mL}$ round-bottom flask under continuous vigorous stirring, and then $5.6 \mathrm{~mL}$ of $1 \%$ sodium citrate was quickly added into the solution. The mixture was further heated for $10 \mathrm{~min}$ and stirred for $10 \mathrm{~min}$. The solution was cooled to room temperature and stored at $4{ }^{\circ} \mathrm{C}$ before use. The concentration of AuNPs was estimated to be $17 \mathrm{nM}$.

\subsection{Procedure for detection of OTA}

A normal procedure for OTA detection is as follows: $50 \mu \mathrm{L}$ of $500 \mathrm{nM}$ aptamer solutions and $50 \mu \mathrm{L}$ of $17 \mathrm{nM}$ AuNPs solution were mixed and incubated for $15 \mathrm{~min}$. Then, $50 \mu \mathrm{L}$ aliquots of different concentrations of OTA were added into the mixture, and incubated for $30 \mathrm{~min}$ at room temperature. Subsequently, $50 \mu \mathrm{L}$ of $170 \mathrm{mM} \mathrm{NaCl}$ was added into the above solution and incubated for $8 \mathrm{~min}$. Finally, the mixed solution was diluted with $\mathrm{H}_{2} \mathrm{O}$ to $500 \mu \mathrm{L}$. The fluorescence intensity of FAM-Apt at $520 \mathrm{~nm}$ was monitored with the excitation wavelength of $475 \mathrm{~nm}$.

\section{Results and discussion}

\subsection{Principle of fluorescence detection}

Fig. 1 shows the analytical process for OTA detection, which makes use of the recognition of aptamer and the quenching of

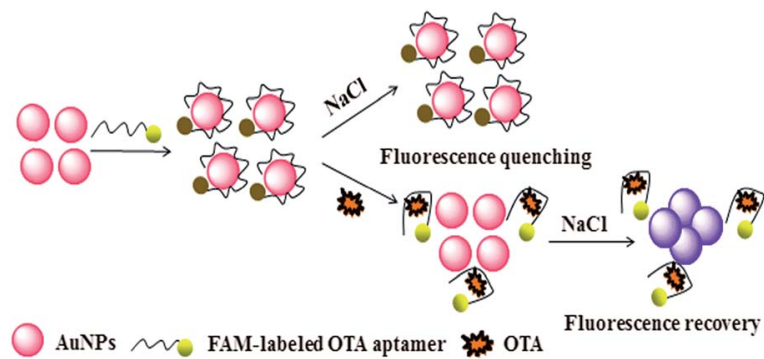

Fig. 1 Schematic illustration of the fluorescence detection of OTA utilizing OTA's aptamer and unmodified AuNPs.

AuNPs. Aptamers are single-stranded oligonucleotides (ssDNA) selected in vitro through SELEX (systematic evolution of ligands by exponential enrichment), ${ }^{21,22}$ and they have been widely used in the detection of proteins, ${ }^{23-28} \mathrm{DNA},{ }^{29}$ and small molecules ${ }^{30-32}$ due to their recognition properties. ${ }^{33}$ The ssDNA aptamers can uncoil sufficiently to expose their bases, and can be adsorbed rapidly on the bare colloidal AuNPs. ${ }^{34}$ Thus, ssDNA can absorb onto the surface through electrostatic attraction. At high salt conditions, the electrostatic repulsion between negatively charged AuNPs is screened, resulting in the aggregation of AuNPs. However, the absorption of ssDNA can prevent the AuNPs against aggregation at high salt concentrations. ${ }^{35}$

Herein, FAM-Apt aptamers are used which easily absorb onto the surface of AuNPs and help to enhance the AuNPs-stability against salt-induced aggregation. The dispersed AuNPs showed superior quenching effect and the fluorescence intensity was obviously decreased (Fig. 2c). However, once OTA was introduced, the conformation of FAM-Apt changed due to specific binding with OTA, thus, the FAM-Apt was scarcely absorbed onto the surface of AuNPs, leading to the recovery of fluorescence (Fig. 2b). When $\mathrm{NaCl}$ was added, the stability of AuNPs decreased under high-salt conditions, resulting in aggregation. Because the quenching of aggregated AuNPs was lower than that of the dispersed AuNPs, the fluorescence showed great recovery (Fig. 2a). The possible mechanism for this fluorescence-quenching based assay could be the suitable matching of the optical properties of AuNPs and FAM-Apt. The dispersed AuNPs have strong absorption at $520 \mathrm{~nm}$, while the FAM-Apt molecules have strong emission at $520 \mathrm{~nm}$. Thus, there is considerable overlap between the

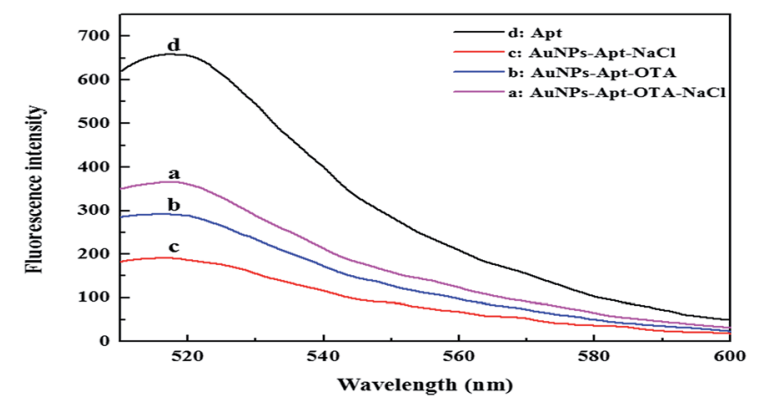

Fig. 2 Fluorescence spectra of FAM-Apt in the different systems. Experimental conditions: AuNPs, $17 \mathrm{nM}$; FAM-Apt, $0.5 \mu \mathrm{M}$; OTA, $1 \mu \mathrm{M}$; $\mathrm{NaCl}, 17 \mathrm{mM}$. 


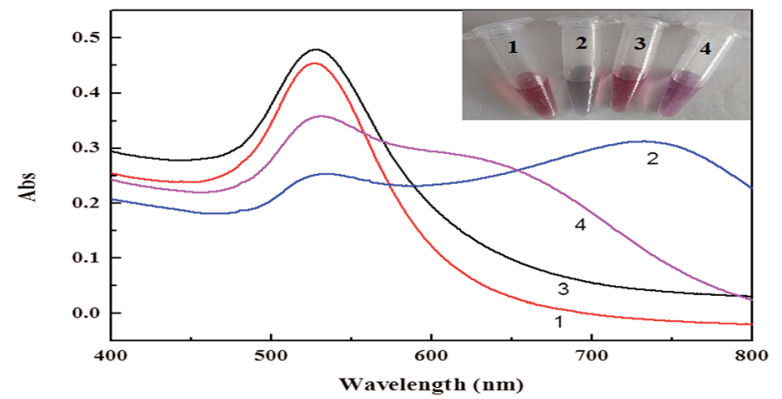

Fig. 3 UV-Vis spectra of AuNPs solution in the different systems. (1) AuNPs, (2) AuNPs + NaCl, (3) AuNPs + FAM-Apt + NaCl (4) AuNPs + FAM-Apt + OTA + NaCl. Experimental conditions: AuNPs, 17 nM; FAMApt, $0.5 \mu \mathrm{M}$; OTA, $1 \mu \mathrm{M} ; \mathrm{NaCl}, 17 \mathrm{mM}$.

absorption spectra of AuNPs and the emission spectra of FAM-Apt. As a result, fluorescence resonance energy transfer (FRET) can occur between AuNPs and FAM-Apt, which leads to the quenching of FAM-Apt. On the other hand, dispersed AuNPs have high surface-to-volume ratio, but when the AuNPs are aggregated, their surface-to-volume ratio decreases greatly. Therefore, the number of FAM-Apt molecules absorbed on the surface of aggregated AuNPs decreases, which causes the FRET between AuNPs and FAM-Apt to weaken. Therefore, the fluorescence intensity increased. Thereby, the fluorescence intensity provided a good means to conduct the quantitative analysis of OTA.

Fig. 3 shows the UV-Vis spectra of the AuNPs solution under different experimental conditions. As seen from Fig. 3, there was a maximum absorption peak at $520 \mathrm{~nm}$ for the AuNPs, which is attributed to the typical plasmon band of the nanoscale $\mathrm{Au}$ (Fig. 3, curve 1). When the $\mathrm{NaCl}$ salt was added to the AuNPs, a new peak appeared at about $750 \mathrm{~nm}$. Correspondingly, the color of the AuNPs solution changed from red to blue (Fig. 3, curve 2). When the FAM-Apt was added to this system, the peak at about $520 \mathrm{~nm}$ remained and the color of the AuNPs solution did not change with the addition of salt (Fig. 3, curve 3). Then, following the addition of OTA, FAM-Apt could specifically bind with OTA, and a new peak appeared at about $650 \mathrm{~nm}$ when $\mathrm{NaCl}$ was added. Correspondingly, the color of the AuNPs solution changed from red to purple (Fig. 3, curve 4). These results are consistent with the transmission electron microscopy (TEM) images (Fig. S1†). Fig. S1† shows that the AuNPs were mostly spherical in shape, and their size was uniform with an average diameter of about $13 \mathrm{~nm}$. In the presence of FAM-Apt, AuNPs remained well dispersed in salt solution. When OTA was added, significant aggregation of AuNPs occurred.

\subsection{Optimization of detection conditions}

Selection of appropriate experimental parameters is very important as these factors can affect the results of the entire experiment. In order to obtain the accurate and reproducible results, the different experimental conditions for the detection of OTA were optimized, such as the concentration of $\mathrm{NaCl}$, concentration of FAM-Apt, concentration of AuNPs, reaction time, and $\mathrm{pH}$.

The concentration of $\mathrm{NaCl}$ was firstly studied as it is an important parameter in the assay. Fig. $\mathrm{S} 2 \uparrow$ shows the fluorescence intensity of FAM-Apt with varying $\mathrm{NaCl}$ concentrations. It can be seen that there was low fluorescence intensity at very low $\mathrm{NaCl}$ concentration, because there was not enough $\mathrm{NaCl}$ to aggregate the AuNPs. The fluorescence intensity increased between 0 and $17 \mathrm{mM}$, and then decreased. The reason for this behavior was that the high salt concentration caused the aggregation of AuNPs, which in turn caused the decrease in fluorescence intensity. When the $\mathrm{NaCl}$ concentration was $17 \mathrm{mM}$, the fluorescence intensity was the highest. So the optimized concentration of $\mathrm{NaCl}$ used for the following experiments was $17 \mathrm{mM}$.

The concentration of AuNPs was another important factor to be evaluated. The fluorescence intensity of FAM-Apt gradually decreased with the increase in concentration of AuNPs. However, most of the fluorescence was effectively quenched when the concentration of AuNPs was $8.5 \mathrm{nM}$ (Fig. S3 $\dagger$ ). So the optimized concentration of AuNPs was 8.5 nM. In this work, the appropriate concentration of FAM-Apt was also investigated. As shown in Fig. S4, $\uparrow A_{530}$ is the UV absorbance of the AuNPs at $530 \mathrm{~nm}$. $A_{530}$ of AuNPs gradually increased with the increase in concentration of FAM-Apt. $A_{530}$ of AuNPs tended to be stable when the concentration of FAM-Apt rose to $0.5 \mu \mathrm{M}$, indicating that FAM-Apt concentration of $0.5 \mu \mathrm{M}$ can efficiently protect AuNPs from salt-induced aggregation. Therefore, the optimum concentration of FAM-Apt was determined to be $0.5 \mu \mathrm{M}$ for the following OTA detection.

In this work, the binding time of OTA-FAM-Apt (Fig. S5 $\dagger$ ) and the reaction time of AuNPs- $\mathrm{NaCl}$ (Fig. S6†) also strongly affected the detection results. As seen from Fig. S5, $\dagger$ the fluorescence intensity of FAM-Apt increased with increasing binding time, and reached a maximum at $30 \mathrm{~min}$. As shown in Fig. $\mathrm{S} 6, \uparrow$ the fluorescence intensity of FAM-Apt gradually decreased and tended to level off after the reaction time reached $8 \mathrm{~min}$. Therefore, the appropriate binding time of OTA-FAM-Apt and reaction time of AuNPs-NaCl were determined to be $30 \mathrm{~min}$ and $8 \mathrm{~min}$, respectively. Lastly, pH was also optimized. As shown in Fig. S7, $\dagger$ the fluorescence intensity reached the maximum value when the $\mathrm{pH}$ was 8.5. Therefore, the appropriate $\mathrm{pH}$ was chosen as 8.5.

\subsection{Quantitative measurement of OTA}

Under the optimal conditions, the fluorescence intensity increased as the concentration of OTA increased. As shown in Fig. 4, there was a good linear relationship between the fluorescence restoration $\left(\Delta F, F=F-F_{0}\right.$, where $F$ and $F_{0}$ are the fluorescence intensities of the system at $520 \mathrm{~nm}$ in the presence and absence of OTA, respectively) and the concentration of OTA. The curve showed a good response for OTA at concentrations of 25-300 nM. The regression equation was determined to be $\Delta F=$ $0.293 C+4.263$ ( $C$ is the concentration of OTA, nM) with a correlation coefficient of 0.9957 . The relative standard deviation (RSD) for $100 \mathrm{nM}$ OTA measurement was found to be $2.0 \%$ $(n=11)$. The limit of detection (LOD) was found to be $22.7 \mathrm{nM}$, as calculated by three times the standard deviation of blanks. The linear relationship between fluorescence intensity and OTA concentration, and the low value of LOD determined in this study are comparable to the standard analytical method based on TLC-FD for $25 \mathrm{nM}$ (GB/T, 5009.96-2003, 2003). The obtained sensitivity with this method is better compared to colorimetric 




Fig. 4 Fluorescence emission spectra of OTA-FAM-Apt-AuNPs$\mathrm{NaCl}$ system with the presence of increasing concentrations of OTA $\left(25,50,100,200,300 \mathrm{nmol} \mathrm{L}^{-1}\right)$. Inset: the linear calibration of fluorescence recovery value $\Delta F\left(F-F_{0}\right)$ versus OTA concentration.



Fig. 5 Specificity assays of OTA. The fluorescence of FAM-Apt-AuNPs solution in the presence of $0.5 \mu \mathrm{M}$ OTA and $5 \mu \mathrm{M}$ of other analytes.

methods. ${ }^{13}$ The detection limit of the sensor based on singlewalled carbon nanotubes (SWCNTs) ${ }^{16}$ and graphene oxide $(\mathrm{GO})^{17}$ are $24.1 \mathrm{nM}$ and $21.8 \mathrm{nM}$, respectively. The detection limit in this work is comparable or even better than those previous methods. On the other hand, the advantages of the sensor based on AuNPs are simple operation and low cost compared to SWCNTs and GO. Although the detection limit is not lower than HPLC $^{6}$ and ELISA, ${ }^{6}$ this method is still superior in terms of its simplicity, rapidity and cost saving.

\subsection{Interference test}

To evaluate the selectivity of this method for OTA, several kinds of potential interfering substances were selected as interferences, including ethanol, serine, maltose, starch, and $\mathrm{Na}^{+}$. All these interferences were tested under the same conditions with OTA. As shown in Fig. 5, these interferences had no effects on the fluorescence. However, a significant increase in fluorescence was observed for OTA. The good selectivity of the aptasensor is attributed to the high specificity of the aptamer.

\subsection{Analysis of real samples}

In order to verify the practicability of the method, real food samples including corn flour, beer (Tsingtao) and coffee (Nescafe) were purchased from a local supermarket. For this analysis, $1.0 \mathrm{~g}$ of corn flour was dispersed in methanol and centrifuged at $4000 \mathrm{r}$ for $5 \mathrm{~min}$. Then, the supernatant was
Table 1 Detection of OTA in samples of corn flour, beer and coffee with different amounts of OTA. $(n=3)^{a}$

\begin{tabular}{lclll}
\hline Sample & $\begin{array}{l}\text { Added } \\
\left(\mathrm{nmol} \mathrm{L}^{-1}\right)\end{array}$ & $\begin{array}{l}\text { Found } \\
\left(\mathrm{nmol} \mathrm{L}^{-1}\right)\end{array}$ & $\begin{array}{l}\text { Recovery } \\
(\%)\end{array}$ & $\begin{array}{l}\text { RSD } \\
(\%)\end{array}$ \\
\hline Cornmeal & 0.0 & ND & - & - \\
& 100.0 & 103.9 & 103.9 & 2.1 \\
\multirow{4}{*}{ Beer } & 300.0 & 270.6 & 90.2 & 1.1 \\
& 0.0 & ND & - & - \\
Coffee & 100.0 & 113.2 & 113.2 & 2.6 \\
& 300.0 & 271.8 & 90.6 & 3.5 \\
& 0.0 & 4.8 & - & - \\
& 100.0 & 115.7 & 110.9 & 2.5 \\
& 300.0 & 286.3 & 93.9 & 2.0
\end{tabular}

${ }^{a}$ ND: not detected.

collected and analyzed for the content of OTA by the same method as described above. Beer and coffee were diluted 100fold by deionized water, and then analyzed for OTA content. As shown in Table 1, the obtained recovery ranged from $90.2 \%$ to $113.2 \%$. The experimental results demonstrated that this method can be used to detect OTA in real food samples.

\section{Conclusions}

This paper introduced a novel strategy for the detection and determination of OTA based on the higher quenching effect of dispersed AuNPs compared to aggregated AuNPs. The strategy has several significant advantages: (1) AuNPs do not need to be modified, and multiple centrifugation and separation steps are avoided. (2) This method has good selectivity for OTA with the detection limit of $22.7 \mathrm{nM}$. Taken together, these advantages make this assay simple, robust, inexpensive, and therefore promising for on-site testing of OTA. The simple method has been successfully used for the detection of OTA in real samples.

\section{Acknowledgements}

This work was financially supported by the National Natural Science Foundation of China (No. 20877037) and the Natural Science Foundation of Shandong Province, China (No. ZR2015BL030).

\section{References}

1 S. C. Duarte, A. Pena and C. M. Lino, Crit. Rev. Toxicol., 2011, 41, 187-2122.

2 A. Pfohl-Leszkowicz and R. A. Manderville, Mol. Nutr. Food Res., 2007, 51, 61-99.

3 P. D. Andrade, J. L. Gomes da Silva and E. D. Caldas, $J$. Chromatogr. A, 2013, 1304, 61-68.

4 Y. J. Cho, D. H. Lee, D. O. Kim, W. K. Min, K. T. Bong, G. G. Lee and J. H. Seo, J. Agric. Food Chem., 2005, 53, 8447-8451.

5 X. W. Lai, D. L. Sun, C. Q. Ruan, H. Zhang and C. L. Liu, J. Sep. Sci., 2014, 37, 92-98. 
6 V. Dohnal, V. Dvorák, F. Malír, V. Ostry and T. Roubal, Food Chem. Toxicol., 2013, 62, 427-431.

7 L. Abrunhosa, H. Morales, C. Soares, T. Calado, A. S. VilaCha, M. Pereira and A. Venancio, Crit. Rev. Food Sci. Nutr., 2016, 56, 249-265.

8 Y. Liang, X. Huang, R. Yu, Y. Zhou and Y. Xiong, Anal. Chim. Acta, 2016, 14, 195-201.

9 M. Mozaffari, M. Bayat and A. Mohsenifar, Microchim. Acta, 2016, 183, 3093-3099.

10 D. Moscone, F. Arduini and A. Amine, Methods Mol. Biol., 2011, 739, 217-235.

11 S. K. Ghosh and P. Tarasankar, Chem. Rev., 2011, 107, 47974862.

12 Q. Yuan, D. Lu, X. Zhang, Z. Chen and W. Tan, TrAC, Trends Anal. Chem., 2012, 39, 72-86.

13 C. Yang, Y. Wang, J. L. Marty and X. R. Yang, Biosens. Bioelectron., 2011, 26, 2724-2727.

14 D. Bu, H. S. Zhuang, G. G. Yang and X. X. Ping, Sens. Actuators, B, 2014, 195, 540-548.

15 W. Li, Y. F. Dong, X. Wang, H. Li and D. K. Xu, Biosens. Bioelectron., 2015, 66, 43-49.

16 Z. J. Guo, J. T. Ren, J. H. Wang and E. K. Wang, Talanta, 2011, 85, 2517-2521.

17 L. F. Sheng, J. T. Ren, Y. Q. Miao, J. H. Wang and E. K. Wang, Biosens. Bioelectron., 2011, 26, 3494-3499.

18 Z. F. Gao, W. W. Song, H. Q. Luo and N. B. Li, Biosens. Bioelectron., 2015, 65, 360-365.

19 X. L. Qin, A. G. Xu, L. Liu, W. F. Deng, C. Chen, Y. M. Tan, Y. C. Fu, Q. J. Xie and S. Z. Yao, Chem. Commun., 2015, 51, 8540-8543.
20 H. Z. He, K. H. Leung, W. Wang, D. S. Chan, C. H. Leung and D. L. Ma, Chem. Commun., 2014, 50, 5313-5315.

21 T. Lang, K. Johanning, H. Metzler, S. Piepenbrock, C. Solomon, N. Rahe-Meyer and K. A. Tanaka, Anesth. Analg., 2009, 108, 751-758.

22 H. Zhang, L. Wang and W. Jiang, Talanta, 2011, 85, 725-729.

23 J. A. Cruz-Aguado and G. Penner, J. Agric. Food Chem., 2008, 56, 10456-10461.

24 C. Tuerk and L. Gold, Science, 1990, 249, 505-510.

25 N. Hamaguchi, A. Ellington and M. Stanton, Anal. Biochem., 2011, 294, 126-131.

26 M. Levy, S. F. Cater and A. D. Ellington, ChemBioChem, 2005, 6, 2163-2166.

27 E. Heyduk and T. Heyduk, Anal. Chem., 2005, 77, 1147-1156. 28 R. Yamamotofujita and P. K. Kumar, Anal. Chem., 2005, 77, 5460-5466.

29 V. Pavlov, B. Shlyahovsky and I. Willner, J. Am. Chem. Soc., 2005, 127, 6522-6523.

30 R. P. Fahlman and D. Sen, J. Am. Chem. Soc., 2002, 124, 46104616.

31 X. Tan, S. K. Dey, C. Telmer, X. Zhang, B. A. Armitage and M. P. Bruchez, ChemBioChem, 2014, 15, 205-208.

32 Q. Zhao, Q. Lv and H. Wang, Anal. Chem., 2014, 86, 12381245.

33 Y. Wu, S. Zhan, F. Wang, L. He, W. Zhi and P. Zhou, Chem. Commun., 2012, 48, 4459-4461.

34 H. Li and L. Rothberg, Proc. Natl. Acad. Sci. U. S. A., 2004, 101, 14036-14039.

35 L. Di, A. Wieckowska and I. Willner, Angew. Chem., 2008, 120, 3991-3995. 